\title{
Effect of Progenitor Cells on Myocardial Perfusion and Metabolism in Patients After Recanalization of a Chronically Occluded Coronary Artery
}

\author{
Kai Kendziorra ${ }^{1}$, Henryk Barthel ${ }^{1}$, Sandra Erbs ${ }^{2}$, Frank Emmrich ${ }^{3}$, Rainer Hambrecht ${ }^{2,4}$, Gerhard Schuler $^{2}$, Osama Sabri ${ }^{1}$, \\ and Regine Kluge ${ }^{1}$ \\ ${ }^{1}$ Department of Nuclear Medicine, University of Leipzig, Leipzig, Germany; ${ }^{2}$ Heart Center Leipzig GmbH, University of Leipzig, Leipzig, \\ Germany; ${ }^{3}$ Institute of Clinical Immunology and Transfusion Medicine, University of Leipzig, Leipzig, Germany; and ${ }^{4}$ Heart Center \\ Bremen, Bremen, Germany
}

Even after recanalization of a chronic total coronary occlusion, functional recovery is incomplete and parts of the myocardium remain hypoperfused. In this randomized, placebo-controlled, and double-blinded study, we investigated relative changes in myocardial perfusion and glucose metabolism induced by intracoronary administration of blood-derived circulating progenitor cells (CPCs), compared with the natural course in a control group after recanalization of total coronary occlusion. Methods: After recanalization of total coronary occlusion, 26 patients were randomly assigned to the CPC treatment or placebo group. Regional myocardial perfusion and glucose metabolism were assessed by ${ }^{99 m}$ Tc-tetrofosmin SPECT and ${ }^{18} \mathrm{~F}-\mathrm{FDG}$ PET at baseline (after recanalization of total coronary occlusion) and 3 mo after the administration of $69 \pm 14 \times 10^{6}$ CPCs or cell-free serum, respectively. Segments were classified as "normal," "perfusion-metabolism mismatch" (dysfunctional segments with a 99mTc-tetrofosmin-18F-FDG mismatch), or "scar." Results: In contrast to the placebo group, CPC administration resulted in a significant decrease in the number of segments with a perfusionmetabolism mismatch, from $3.0 \pm 0.5$ to $1.7 \pm 0.6$ segments $(P<$ 0.05 vs. baseline). Of the normal segments at baseline, $2.7 \%$ in the CPC group and $30 \%$ in the placebo group revealed a perfusionmetabolism mismatch at follow-up after 3 mo $(P<0.05$ vs. placebo). Conclusion: Intracoronary administration of CPCs significantly reduces the amount of myocardium with a perfusionmetabolism mismatch and prevents areas with normal perfusion and metabolism after recanalization of total coronary occlusion from becoming dysfunctional during the next 3 mo. These results show that PET and SPECT can be used to monitor the effect of progenitor cells on myocardial integrity. More important, they provide evidence supporting expansion of the use of progenitor cell treatment to chronic coronary artery disease.

Key Words: ischemic heart disease; perfusion-metabolism mismatch; progenitor cells; ${ }^{18} \mathrm{~F}-\mathrm{FDG}$ PET

J Nucl Med 2008; 49:557-563

DOI: 10.2967/jnumed.107.046706

Received Aug. 24, 2007; revision accepted Dec. 20, 2007.

For correspondence or reprints contact: Kai Kendziorra, Department of Nuclear Medicine, University of Leipzig, Stephanstrasse 11, 04103 Leipzig, Germany.

E-mail: kenk@medizin.uni-leipzig.de

COPYRIGHT @ 2008 by the Society of Nuclear Medicine, Inc.
$\mathbf{P}$ atients with total occlusion of a main coronary artery are known to benefit significantly from recanalization with regard to major cardiac events (1), long-term survival (2), improvement of left ventricular function (3), and myocardial flow reserve (4). Nevertheless, functional recovery is incomplete, and parts of the myocardium remain with a perfusionmetabolism mismatch (5-7). However, the underlying mechanisms are not fully understood (7). One speculation is that parts of the myocardium are insufficiently supplied because of persistent paradoxical vasoconstriction of epicardial vessels and microvascular dysfunction (8).

Lately, experimental and clinical studies using progenitor cells have shown functional recovery and improved myocardial perfusion in chronic myocardial ischemia $(9,10)$. To investigate whether progenitor cells also demonstrate such a benefit after recanalization of total coronary occlusion, we conducted a randomized, placebo-controlled, and doubleblinded study with intracoronary administration of bloodderived circulating progenitor cells (CPCs) in patients after successful recanalization of total coronary occlusion. We recently published a paper focusing on the methodology of preparation and administration of the CPCs and inflammatory markers, invasive measurements of coronary endothelial function, and assessment of left ventricular function and wall motion with MRI (11). This previous publication also included an aspect of myocardial perfusion and metabolism data. In this present study, we focused on subtle analyses of relative changes in myocardial perfusion and glucose metabolism induced by CPCs, compared with the natural course in a control group, after successful recanalization of total coronary occlusion.

\section{MATERIALS AND METHODS}

The detailed methods of patient selection and of preparation and administration of the progenitor cells were described in our previous publication (11). Briefly, patients with total coronary occlusion, clinical signs or objective measures of myocardial and local wall motion abnormalities as assessed by left ventricular angiography 
were screened. Total coronary occlusion was defined as an occlusion of a native coronary artery for more than $30 \mathrm{~d}$ with no luminal continuity and with thrombolysis in myocardial infarction (TIMI) flow grade 0 or 1 (12). Recanalization with restoration of TIMI flow grade 3 and a residual stenosis of less than $30 \%$ was considered a technical success. After successful recanalization of total coronary occlusion, patients underwent ${ }^{18} \mathrm{~F}-\mathrm{FDG}$ PET and gated ${ }^{99 \mathrm{~m} T c-t e t r o f o s m i n ~ S P E C T}$ to measure myocardial glucose consumption and perfusion. Subsequently, all patients received filgrastim (300 $\mu \mathrm{g}$ of granulocyte colony-stimulating factor) by subcutaneous injections twice a day over a period of $4 \mathrm{~d}$ to increase the number of CPCs in the blood. To select for CPCs, mononuclear cells were purified out of $400 \mathrm{~mL}$ of venous blood collected from each patient and cultured ex vivo for $4 \mathrm{~d}$ in endothelial-specific medium $(13,14)$. After successful recanalization of total coronary occlusion, 26 patients were randomly assigned to the CPC group (13 patients) or the placebo group (13 patients). At a mean of $10 \pm$ $1 \mathrm{~d}$ after successful recanalization of total coronary occlusion, patients randomized into the $\mathrm{CPC}$ group received intracoronary administration of an average of $69 \pm 14 \times 10^{6} \mathrm{CPCs}$, by repeated infusion into the vessel previously reopened, whereas patients randomized into the placebo group received cell-free serum. Ninety percent of the selected cells showed the typical features of CPCs, that is, binding to lectin and uptake of acetylated low-density lipoprotein. Neither the interventionalist nor the clinical investigators (including the nuclear medicine physician) were aware of the randomization results for the individual patients. ${ }^{18} \mathrm{~F}$-FDG PET

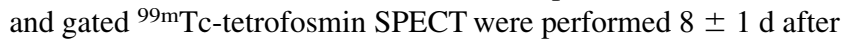
successful recanalization (but before CPC or cell-free serum administration) and 3 mo after administration of CPCs or cell-free serum.

${ }^{18}$ F-FDG PET was performed using a full-ring ECAT EXACT HR + PET scanner (Siemens/CTI). Nondiabetic patients received a single dose of $250 \mathrm{mg}$ of acipimox (Olbemox; Pharmacia Upjohn) after an overnight fast. Sixty minutes later, $50 \mathrm{~g}$ of glucose were given orally. A mean dose of $370 \mathrm{MBq}$ of ${ }^{18} \mathrm{~F}-\mathrm{FDG}$ was injected intravenously, and PET images were acquired in 2-dimensional mode $1 \mathrm{~h}$ later during $30 \mathrm{~min}$, including a 10 -min transmission scan. Diabetic patients ( $n=2$ of the placebo group and $n=1$ of the CPC group) underwent a standard hyperinsulinemic euglycemic clamp, and ${ }^{18} \mathrm{~F}$-FDG was administered when glucose plasma levels started to decrease. The images were reconstructed with filtered backprojection using a Hann filter (full width at half maximum, $7.9 \mathrm{~mm}$ ).

To acquire the perfusion scans, we injected $400 \mathrm{MBq}$ of ${ }^{99 \mathrm{~m}} \mathrm{Tc}-$ tetrofosmin while the patients were resting and after they had fasted overnight. Patients were encouraged to have a fatty meal (including $250 \mathrm{~mL}$ of milk) $15 \mathrm{~min}$ after injection, followed by $500 \mathrm{~mL}$ of water $45 \mathrm{~min}$ later. Image acquisition began $1 \mathrm{~h}$ after injection using a dual-head SPECT camera (Vertex camera; ADAC Laboratories) with detectors positioned at an angle of $90^{\circ}$ and equipped with ultrahigh-resolution collimators and two ${ }^{153} \mathrm{Gd}$ line sources for attenuation measurement. Sixteen views per detector were recorded over a period of $80 \mathrm{~s}$ each, scanning from the $45^{\circ}$ left posterior oblique to the $45^{\circ}$ right anterior oblique positions. Images were recorded in gated mode with simultaneous electrocardiogram recording. The image data underwent standard reconstruction using an iterative algorithm with attenuation and scatter correction. Electrocardiogram-gated data were used to calculate regional wall motion, regional wall thickening, and functional parameters (QGS software; Cedars-Sinai Medical Center).
According to the recommended standardized nomenclature, the left ventricular myocardium was divided into 17 segments (15). The segments were assigned to the territories of the 3 major coronary arteries as recommended. Despite tremendous intersubject variability in the coronary artery blood supply to myocardial segments, there is consensus in the literature that it is appropriate to assign individual segments to specific coronary artery territories (16). The target region was classified as the region corresponding to the reopened chronic coronary occlusion (including segments in the border zone). Cerqueira et al. have provided a detailed description of segment assignment (15). The segment with the highest ${ }^{99 \mathrm{~m}} \mathrm{Tc}-$ tetrofosmin uptake outside the target was designated the reference area. All other segments in the ${ }^{99 \mathrm{~m}} \mathrm{Tc}$-tetrofosmin and ${ }^{18} \mathrm{~F}-\mathrm{FDG}$ studies were adjusted to this reference area by applying a computerized automatic procedure. In the ${ }^{99 \mathrm{~m}} \mathrm{Tc}$-tetrofosmin perfusion and ${ }^{18}$ F-FDG metabolism studies, relative radiotracer uptake in the segments was calculated at baseline and at follow-up after 3 mo.

The segments were classified into 3 categories based on the local ${ }^{99} \mathrm{~m}$ Tc-tetrofosmin and ${ }^{18} \mathrm{~F}-\mathrm{FDG}$ uptake. Segments with normal ${ }^{99 \mathrm{~m}} \mathrm{Tc}$-tetrofosmin uptake and normal ${ }^{18} \mathrm{~F}-\mathrm{FDG}$ uptake $(>80 \%$ of reference) were considered normal. Segments with a perfusion defect (normalized ${ }^{99 \mathrm{~m}} \mathrm{Tc}$-tetrofosmin uptake $<80 \%$ ) were classified as perfusion-metabolism mismatch when a ${ }^{99 \mathrm{~m}} \mathrm{Tc}$-tetrofosmin${ }^{18} \mathrm{~F}-\mathrm{FDG}$ mismatch (increase in ${ }^{18} \mathrm{~F}-\mathrm{FDG}$ uptake $\geq 10 \%$, compared with ${ }^{99 m}$ Tc-tetrofosmin uptake) and a wall motion reduction $(<50 \%$ of maximum on the electrocardiogram-gated images) were present. Segments with a perfusion defect were classified as scar tissue when a ${ }^{99 \mathrm{~m}}$ Tc-tetrofosmin- ${ }^{18} \mathrm{~F}$-FDG match was present $(<10 \%$ difference in ${ }^{99 \mathrm{~m}} \mathrm{Tc}$-tetrofosmin and ${ }^{18} \mathrm{~F}$-FDG uptake). Statistical analysis was performed using the SPSS software package, version 14.0 (SPSS Inc.). The Wilcoxon rank test was performed to detect significant differences between baseline and follow-up. The $\chi^{2}$ test was used to detect group differences. The significance level was set at a $P$ value of less than 0.05 .

\section{RESULTS}

A detailed report on the demographic, clinical, and angiographic data analysis; safety and side-effects of granulocyte colony-stimulating factor stimulation; and clinical followup, assessment of coronary endothelial function, and assessment of global left ventricular function parameters by MRI was given in our previous publication (11). Patients in the placebo group and patients receiving CPC did not significantly differ in demographic, clinical, and angiographic parameters or medication. Four patients ( 2 each from the placebo and CPC groups) did not undergo follow-up perfusion or metabolism imaging studies, because they withdrew their consent. Furthermore, 1 patient in the placebo group was excluded from the study because of reocclusion of the initially successfully reopened total coronary occlusion at the time of serum administration. In 1 patient in the placebo group, the ${ }^{18} \mathrm{~F}-\mathrm{FDG}$ PET images could not be analyzed quantitatively because of a spillover of radioactivity from the intestine. Therefore, the complete follow-up could be performed only on 11 patients from the CPC group and 9 patients randomized to the placebo group. Initially, the CPC group comprised 90 segments in the target region and the placebo group 66. In the baseline studies, in the CPC and placebo 
groups 20 and 9 segments, respectively, were classified as scar; 33 and 24, respectively, as perfusion-metabolism mismatch; and 37 and 33, respectively, as normal.

\section{Time-Dependent Changes in Segments Classified as Normal}

At baseline investigation, that is, after recanalization but before the administration of CPCs or cell-free serum, patients in the CPC and placebo groups were found to have no differences in the mean number of normal segments within the target area. Compared with the baseline situation, at 3 mo the number of normal segments was significantly higher in the CPC group within the target area. This was not the case in the placebo group (Figs. 1 and 2; Table 1).

Follow-up revealed that $94.6 \%$ of the segments classified as normal on the baseline scans remained normal within the target area in the CPC group (2.7\% turned into scar and $2.7 \%$ developed a perfusion-metabolism mismatch), whereas in the placebo group $69.7 \%$ stayed normal and $30.3 \%$ revealed a perfusion-metabolism mismatch (Fig. 3A). These group differences in the fate at follow-up of segments that had been normal on the baseline scans were significant $(P=0.004$, $\chi^{2}$ test).

\section{Time-Dependent Changes in Segments with a Perfusion-Metabolism Mismatch}

Initial results on the fate of segments with a perfusionmetabolism mismatch on the baseline scans were presented in our previous publication (11). At baseline, the mean number of segments with a perfusion-metabolism mismatch within the target area did not differ between patients in the CPC and placebo groups. Compared with the baseline situation, the number of segments with a perfusion-metabolism mismatch at 3 mo was significantly lower in the CPC group within the target area. This was not the case in the placebo group (Table 1).

Follow-up revealed that $48.5 \%$ of the segments displaying a perfusion-metabolism mismatch on the baseline scans became normal within the target area in the CPC group (9.1\% turned into scar and $42.4 \%$ remained with a perfusionmetabolism mismatch), whereas in the placebo group $12.5 \%$ became normal, $66.7 \%$ remained with a perfusion-metabolism mismatch, and $20.8 \%$ turned into scar (Figure 3B). These group differences in the fate at follow-up of segments that had shown a perfusion-metabolism mismatch on the baseline scans were significant $\left(P=0.004, \chi^{2}\right.$ test $)$.

\section{Time-Dependent Changes in Segments Classified as Scar}

The mean number of regions classified as scar did not change significantly in either the CPC or the placebo group (Table 1).

\section{Changes in ${ }^{99 m}$ Tc-Tetrofosmin Uptake}

The relative ${ }^{99 \mathrm{~m}}$ Tc-tetrofosmin uptake increased slightly but significantly from $75 \% \pm 15 \%$ (mean \pm SD) to $78 \% \pm$ $16 \%$ in the 90 segments within the target area after the infusion of CPCs $(P=0.02$, Wilcoxon rank test). In segments of the placebo group, no relative change in ${ }^{99 \mathrm{~m}} \mathrm{Tc}$-tetrofosmin uptake was observed $(74 \% \pm 18 \%$ at baseline and $74 \% \pm$ $17 \%$ after $3 \mathrm{mo}$ ). Of the 55 segments with reduced ${ }^{99 \mathrm{~m}} \mathrm{Tc}-$ tetrofosmin uptake at baseline, 33\% (18 segments) showed normal ${ }^{99 \mathrm{~m} T c-t e t r o f o s m i n}$ uptake at follow-up in the CPC group. In the placebo group, of the 32 segments with reduced ${ }^{99 \mathrm{~m}}$ Tc-tetrofosmin uptake, $12 \%$ (4 segments) demonstrated a

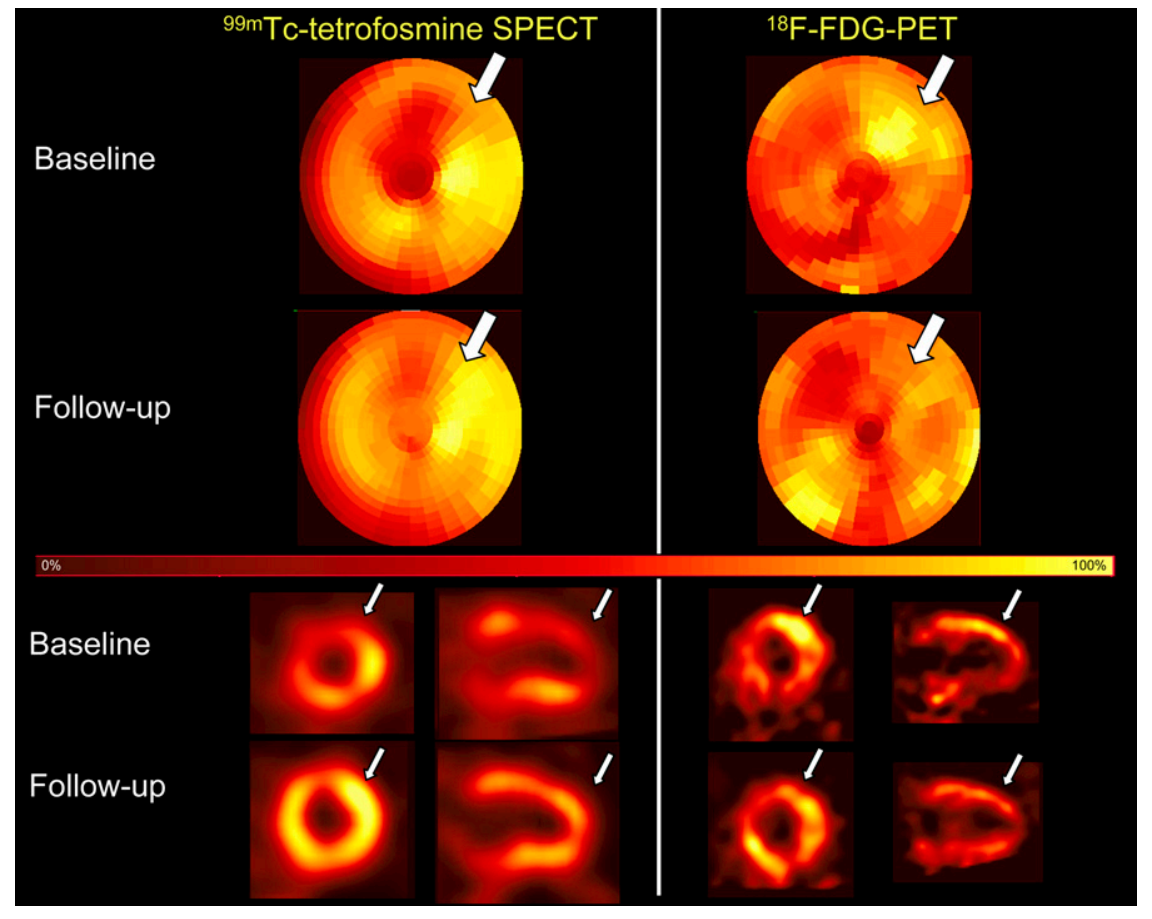

FIGURE 1. 99mTc-Tetrofosmin SPECT and ${ }^{18}$ F-FDG PET studies (bull's-eye view and representative vertical long-axis and short-axis slices) of patient after recanalization of totally occluded left anterior descending coronary artery (baseline) and 3 mo after administration of CPCs (follow-up). Arrows indicate area with reduced $99 \mathrm{~m}$ Tc-tetrofosmin and enhanced ${ }^{18} \mathrm{~F}-\mathrm{FDG}$ uptake corresponding to perfusion-metabolism mismatch in anterolateral area at baseline (arrows) and disappearance of this finding at follow-up. 


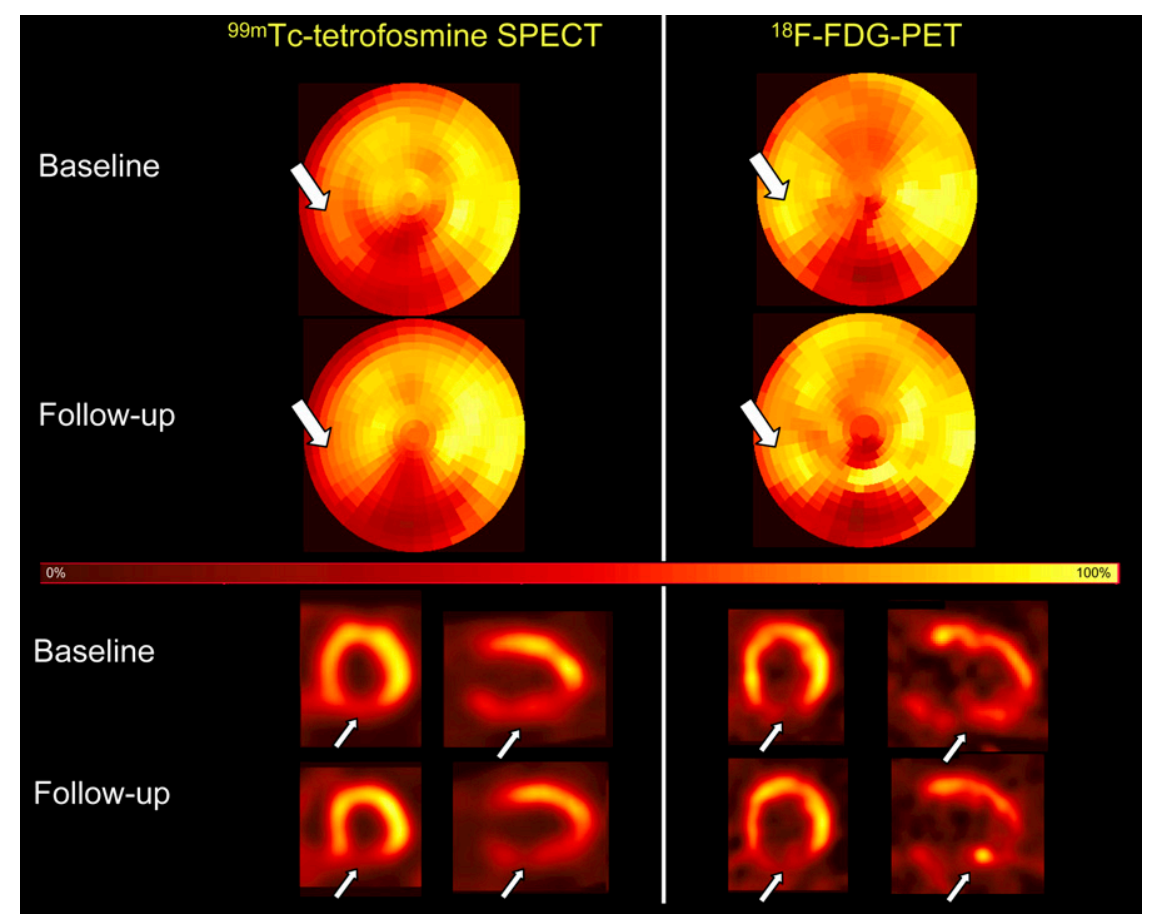

FIGURE 2. $99 \mathrm{mTC}$-Tetrofosmin SPECT and ${ }^{18} \mathrm{~F}-\mathrm{FDG}$ PET studies (bull's-eye view and representative vertical long-axis and short-axis slices) of patient after recanalization of totally occluded right coronary artery (baseline) and 3 mo after administration of cell-free serum (follow-up). Arrows indicate area with reduced 99mTc-tetrofosmin and enhanced ${ }^{18} \mathrm{~F}-\mathrm{FDG}$ uptake corresponding to perfusionmetabolism mismatch in posteroseptal area at baseline. In this area, slight deterioration in perfusion occurs at follow-up.

normal ${ }^{99 \mathrm{~m}} \mathrm{Tc}$-tetrofosmin uptake at follow-up. These group differences in the relative ${ }^{99 \mathrm{~m}}$ Tc-tetrofosmin uptake were significant $\left(P=0.036, \chi^{2}\right.$ test). However, because of the limited number of patients and the interindividual variations, an analysis of these changes on a per-patient basis did not reach the significance level.

The changes in the number of segments classified as normal, perfusion-metabolism mismatch, and scarred between baseline and follow-up within the target area are plotted individually on a per-patient base for the CPC and placebo groups in Figure 4. Normal segments increased and segments with perfusion-metabolism mismatch decreased in most CPC patients.

\section{DISCUSSION}

Our study demonstrated that, compared with the natural course after recanalization of total coronary occlusion, administration of CPCs after recanalization brought about significant differences in perfusion and glucose metabolism in the myocardial target area.

Even after successful recanalization of total coronary occlusion, parts of the myocardium remain nonfunctional, with a hypoperfusion-metabolism mismatch (6). This was the case in $36 \%$ of the segments in the target area in our study at $8 \pm 1 \mathrm{~d}$ after recanalization, whereas $45 \%$ showed normal perfusion at that time. In the time until the 3-mo follow-up, $30 \%$ of normal segments in the recanalized area developed a perfusion-metabolism mismatch in the group that had not been given progenitor cells. This deterioration could be attributable to disturbances in microvascular circulation or insufficient blood flow due to persistent paradoxical vasoconstriction of epicardial vessels (8). Because only patients with a successful recanalization (TIMI flow grade 3 with a residual stenosis of $<30 \%$ ) were included in the study and no flow-limiting in-stent restenosis occurred (11), the deterioration in perfusion and development of a perfusionmetabolism mismatch in the placebo group was likely due

TABLE 1

Mean Number of Segments Within Target Area

\begin{tabular}{|c|c|c|c|c|c|c|}
\hline \multirow[b]{2}{*}{ Segment type } & \multicolumn{3}{|c|}{ Placebo group } & \multicolumn{3}{|c|}{ CPC group } \\
\hline & Baseline & Follow-up & $P$ & Baseline & Follow-up & $P$ \\
\hline Normal & $3.7(0.9)$ & $3.0(0.8)$ & NS & $3.4(0.5)$ & $5.2(0.9)$ & 0.015 \\
\hline Mismatch & $2.7(0.6)$ & $3.6(0.6)$ & NS & $3.0(0.5)$ & $1.7(0.6)$ & 0.048 \\
\hline Scar & $1.0(0.4)$ & $0.8(0.5)$ & NS & $1.8(0.5)$ & $1.3(0.6)$ & NS \\
\hline
\end{tabular}



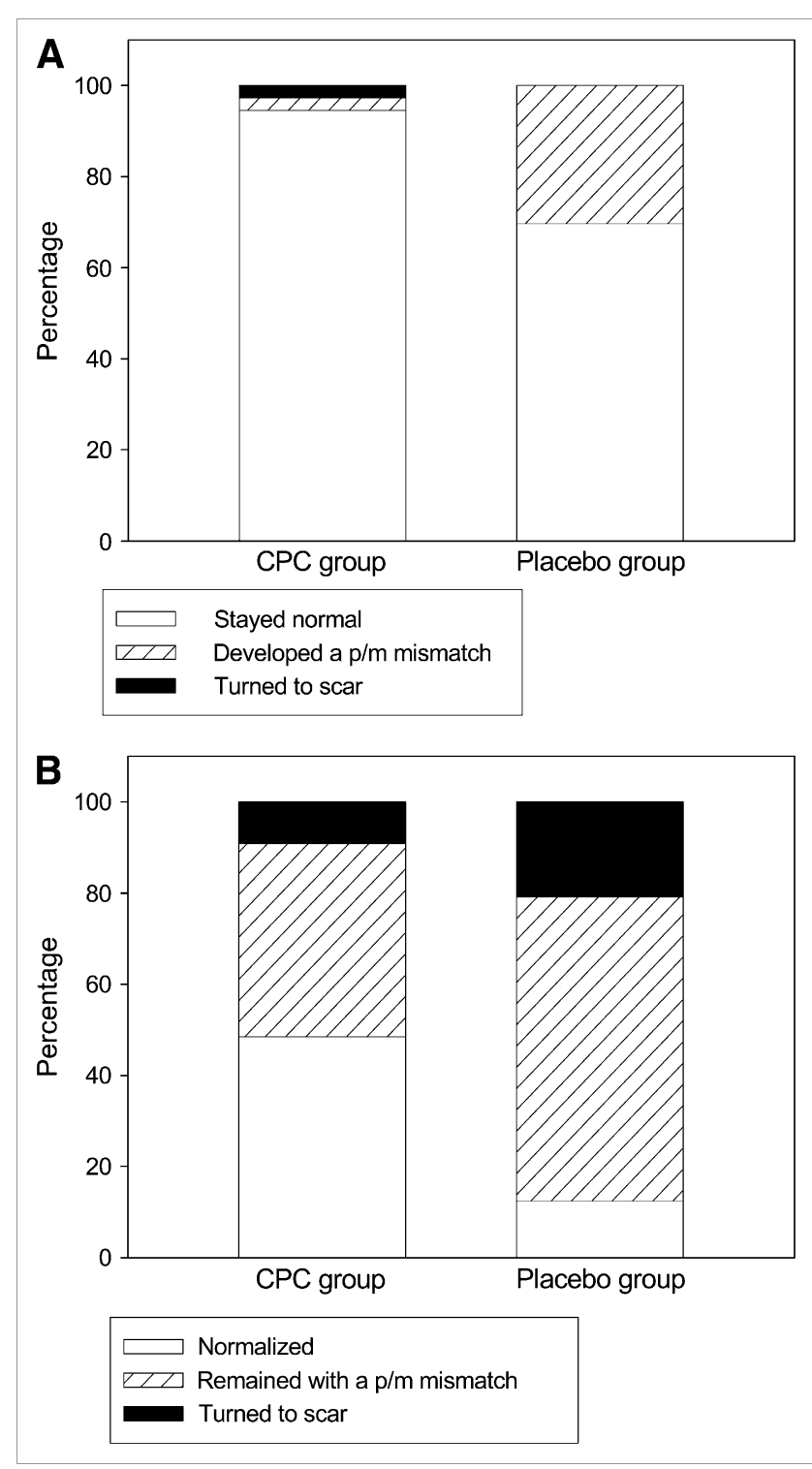

FIGURE 3. Three-month follow-up of segments classified as normal $(A)$ or having perfusion-metabolism $(\mathrm{p} / \mathrm{m})$ mismatch $(B)$ within area of total coronary occlusion (target area) at $8 \pm 1 \mathrm{~d}$ after recanalization. For both situations, group differences were significant $\left(P<0.05, \chi^{2}\right.$ test $)$.

to disturbances in microvascular circulation. We would hypothesize that the observed changes in perfusion rather reflect the natural time course after reopening of total coronary occlusion, with a short-term improvement (day 8) and subsequent deterioration ( $3 \mathrm{mo}$ ) due to disturbances in microcirculation in the placebo group. To our knowledge, no studies have investigated changes in perfusion and metabolism in a comparable interval shortly after recanalization of total coronary occlusion. Bax et al. performed follow-up studies 3 and 14 mo after surgical revascularization (17) and demonstrated a reduction of dysfunctional segments, with perfusion-metabolism mismatch in the later course. However, the situation immediately after recanalization is un-

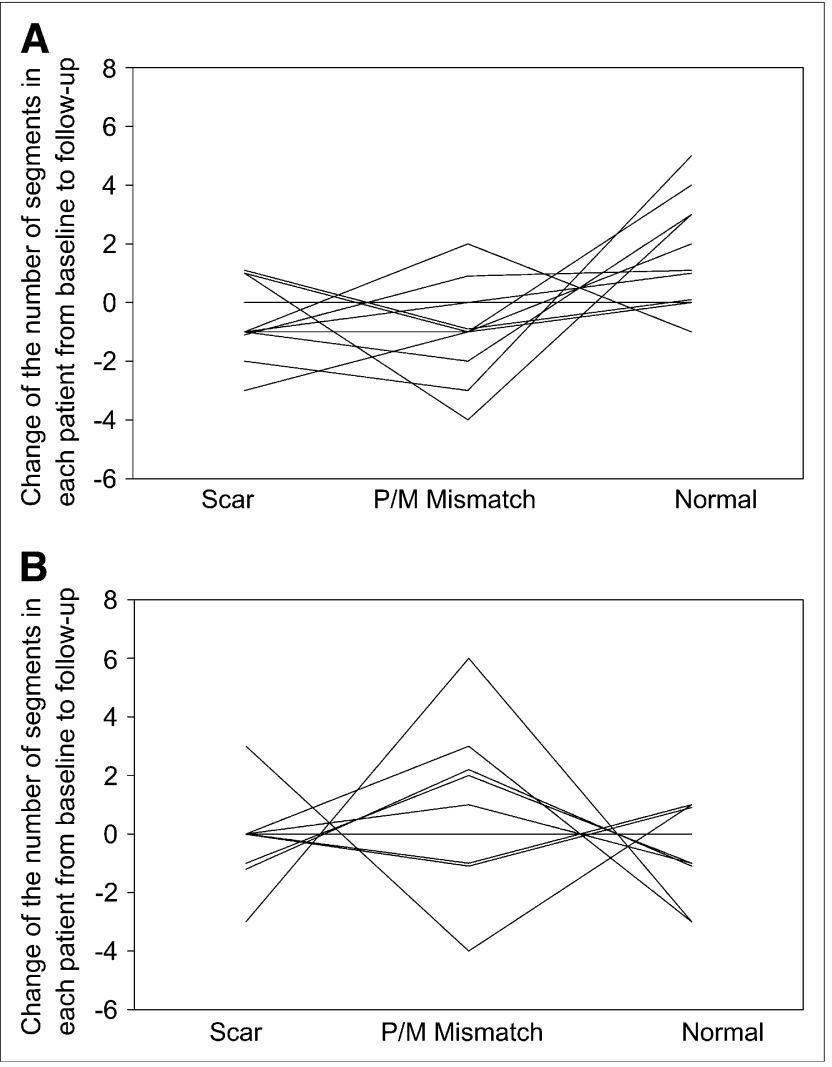

FIGURE 4. Change in number of segments classified as scar, perfusion-metabolism $(\mathrm{p} / \mathrm{m})$ mismatch, or normal in each patient from baseline to follow-up, for CPC group (A) and placebo group (B). Each patient is represented by 1 line. In most CPC patients, normal segments increase and segments with perfusionmetabolism mismatch decrease. Table 1 provides the statistics.

known, and the percentage of scarred tissue 3 mo after revascularization in their study was significantly higher than in our patients (55\% vs. $10 \%$, respectively).

Intracoronary infusion of CPCs after successful recanalization of total coronary occlusion not only led to a recovery of relative myocardial perfusion over $3 \mathrm{mo}$ and a normalization of myocardial glucose metabolism in a part of the target area but also prevented myocardium with normal perfusion and glucose metabolism early after recanalization of total coronary occlusion from developing a perfusion-metabolism mismatch during the next 3 mo. Normalization of glucose metabolism and myocardial perfusion was seen in nearly $50 \%$ of the segments with an initial perfusion-metabolism mismatch within the target area. A significantly higher percentage of segments initially classified as normal stayed normal in the CPC than in the placebo group.

Various studies have suggested an improvement in clinical symptoms and myocardial stress perfusion by progenitor cell transplantation in patients with chronic ischemic heart disease $(10,18-20)$. In these studies, progenitor cells were administered without a revascularization procedure and mainly by transendocardial injection. An animal study 
assessing the effect of the sole administration of bone marrow-derived progenitor cells after chronic myocardial infarction without recanalization procedures detected a greater improvement in myocardial perfusion or even normalization of perfusion by ${ }^{99 \mathrm{~m}} \mathrm{Tc}$-sestamibi pinhole SPECT if perfusion defects were less severe in the initial stage (21). Improvement was maximal in segments with a significant residual level of perfusion, such as those in the border zone of myocardial infarction. Because this area is where a mismatch in perfusion and metabolism mainly occurs, this finding does indeed argue for beneficial effects of progenitor cells in the state of single-vessel total coronary occlusion, where perfusion is at least partially obtained-i.e., because of collateral vessels (22). The effect of progenitor cells could be due to normalization of endothelial function and the subsequently improved microvascular and macrovascular function. Additionally, an advancement of endothelium-dependent vasodilatation due to the reconstitution of damaged endothelium by CPCs $(23,24)$ or the release of survival factors by CPCs and thus the promotion of survival and proliferation of resident endothelial cells within the epicardial coronary arteries by those cytokines (25) has been supposed.

Our study found no significant changes in scarred segments independent of the administration of progenitor cells. This finding tallies with other progenitor cell therapy studies of chronic ischemia. In a small study including 7 patients, Tossios et al. (26) noted no significant improvement in myocardial viability after the concurrent transplantation of bone marrow-derived progenitor cells in patients undergoing coronary artery bypass surgery. In the study by Beeres et al. (10), the percentage of myocardial segments with some extent of scarring remained unchanged at the 3-mo followup. However, some investigators found a significant increase in myocardial viability in nonacute infarction $3 \mathrm{mo}$ after surgery and progenitor cell administration (27).

One limitation of our study is the lack of perfusion and metabolism data before the recanalization of total coronary occlusion. However, because a considerable percentage of recanalization fails in total coronary occlusion, successful recanalization of total coronary occlusion was a prerequisite for randomization into the placebo or CPC group. It would be of interest to analyze the course of perfusion and metabolism changes from the initial situation of chronic ischemia before recanalization.

Our data might in principle be compromised by the fact that myocardial perfusion was studied by a SPECT tracer whereas glucose metabolism was measured by PET. However, because of the correction for measurement attenuation in the SPECT study, the influence of attenuation effects ought to be minimized (28), thus providing valid data for a segmental analysis.

Despite randomization, localization of the total coronary occlusion differed significantly between the CPC and placebo groups. The possibility that this difference affected our results cannot be ruled out.

\section{CONCLUSION}

Intracoronary administration of CPCs after successful recanalization of total coronary occlusion leads to a treatment-specific normalization of myocardium with a perfusion-metabolism mismatch resulting in a reduction of myocardium at risk. In addition, CPCs seem to have a positive effect on myocardial areas with normal perfusion and metabolism shortly after recanalization of total coronary occlusion, thus preventing the redevelopment of a perfusionmetabolism mismatch during the next few months. This prolonged effect suggests a slowing of disease progression due to CPCs. Further longitudinal studies should address whether both effects reduce the risk of cardiac events over longer periods.

\section{REFERENCES}

1. Lamas GA, Flaker GC, Mitchell G, et al. Effect of infarct artery patency on prognosis after acute myocardial-infarction. Circulation. 1995;92:11011109

2. Suero JA, Marso SP, Jones PG, et al. Procedural outcomes and long-term survival among patients undergoing percutaneous coronary intervention of a chronic total occlusion in native coronary arteries: a 20-year experience. J Am Coll Cardiol. 2001;38:409-414.

3. Sirnes PA, Myreng Y, Molstad P, Bonarjee V, Golf S. Improvement in left ventricular ejection fraction and wall motion after successful recanalization of chronic coronary occlusions. Eur Heart J. 1998;19:273-281.

4. Fath-Ordoubadi F, Beatt KJ, Spyrou N, Camici PG. Efficacy of coronary angioplasty for the treatment of hibernating myocardium. Heart. 1999;82:210-216.

5. Schwarz ER, Schoendube FA, Kostin S, et al. Prolonged myocardial hibernation exacerbates cardiomyocyte degeneration and impairs recovery of function after revascularization. J Am Coll Cardiol. 1998;31:1018-1026.

6. Vanoverschelde JL, Depre C, Gerber BL, et al. Time course of functional recovery after coronary artery bypass graft surgery in patients with chronic left ventricular ischemic dysfunction. Am J Cardiol. 2000;85:1432-1439.

7. Wijns W, Vatner SF, Camici PG. Hibernating myocardium. N Engl J Med. 1998;339:173-181.

8. Werner GS, Ferrari M, Richartz BM, Gastmann O, Figulla HR. Microvascular dysfunction in chronic total coronary occlusions. Circulation. 2001;104:11291134 .

9. Kocher AA, Schuster MD, Szabolcs MJ, et al. Neovascularization of ischemic myocardium by human bone-marrow-derived angioblasts prevents cardiomyocyte apoptosis, reduces remodeling and improves cardiac function. Nat Med. 2001;7:430-436.

10. Beeres SLMA, Bax JJ, Dibbets P, et al. Effect of intramyocardial injection of autologous bone marrow-derived mononuclear cells on perfusion, function, and viability in patients with drug-refractory chronic ischemia. $J$ Nucl Med. 2006;47: 574-580.

11. Erbs S, Linke A, Adams V, et al. Transplantation of blood-derived progenitor cells after recanalization of chronic coronary artery occlusion: first randomized and placebo-controlled study. Circ Res. 2005;97:756-762.

12. Olivari Z, Rubartelli P, Piscione F, et al. Immediate results and one-year clinical outcome after percutaneous coronary interventions in chronic total occlusions: data from a multicenter, prospective, observational study (TOAST-GISE). J Am Coll Cardiol. 2003;41:1672-1678.

13. Adams V, Lenk K, Linke A, et al. Increase of circulating endothelial progenitor cells in patients with coronary artery disease after exercise-induced ischemia. Arterioscler Thromb Vasc Biol. 2004;24:684-690.

14. Asahara T, Murohara T, Sullivan A, et al. Isolation of putative progenitor endothelial cells for angiogenesis. Science. 1997;275:964-967.

15. Cerqueira MD, Weissman NJ, Dilsizian V, et al. Standardized myocardial segmentation and nomenclature for tomographic imaging of the heart: a statement for healthcare professionals from the Cardiac Imaging Committee of the Council on Clinical Cardiology of the American Heart Association. Circulation. 2002;105: 539-542.

16. Gallik DM, Obermueller SD, Swarna US, et al. Simultaneous assessment of myocardial perfusion and left ventricular function during transient coronary occlusion. J Am Coll Cardiol. 1995;25:1529-1538. 
17. Bax JJ, Visser FC, Poldermans D, et al. Time course of functional recovery of stunned and hibernating segments after surgical revascularization. Circulation. 2001;104:I314-I318.

18. Perin EC, Dohmann HFR, Borojevic R, et al. Transendocardial, autologous bone marrow cell transplantation for severe, chronic ischemic heart failure. Circulation. 2003;107:2294-2302.

19. Perin EC, Dohmann HFR, Borojevic R, et al. Improved exercise capacity and ischemia 6 and 12 months after transendocardial injection of autologous bone marrow mononuclear cells for ischemic cardiomyopathy. Circulation. 2004;110:II213-II218.

20. Fuchs S, Satler LF, Kornowski R, et al. Catheter-based autologous bone marrow myocardial injection in no-option patients with advanced coronary artery disease: a feasibility study. J Am Coll Cardiol. 2003;41:1721-1724.

21. Tran N, Franken PR, Maskali F, et al. Intramyocardial implantation of bone marrow-derived stem cells enhances perfusion in chronic myocardial infarction: dependency on initial perfusion depth and follow-up assessed by gated pinhole SPECT. J Nucl Med. 2007;48:405-412.

22. Aboul-Enein F, Kar S, Hayes SW, et al. Influence of angiographic collateral circulation on myocardial perfusion in patients with chronic total occlusion of a single coronary artery and no prior myocardial infarction. J Nucl Med. 2004; 45:950-955.
23. Werner N, Junk S, Laufs U, et al. Intravenous transfusion of endothelial progenitor cells reduces neointima formation after vascular injury. Circ Res. 2003; 93:E17-E24.

24. Higashi Y, Kimura M, Hara K, et al. Autologous bone-marrow mononuclear cell implantation improves endothelium-dependent vasodilation in patients with limb ischemia. Circulation. 2004;109:1215-1218.

25. Rehman J, Li JL, Orschell CM, March KL. Peripheral blood "endothelial progenitor cells" are derived from monocyte/macrophages and secrete angiogenic growth factors. Circulation. 2003;107:1164-1169.

26. Tossios P, Muller-Ehmsen J, Schmidt M, et al. No evidence of myocardial restoration following transplantation of mononuclear bone marrow cells in coronary bypass grafting surgery patients based upon cardiac SPECT and ${ }^{18} \mathrm{~F}$ PET. BMC Med Imaging. 2006;6:7.

27. Herreros J, Prosper F, Perez A, et al. Autologous intramyocardial injection of cultured skeletal muscle-derived stem cells in patients with non-acute myocardial infarction. Eur Heart J. 2003;24:2012-2020.

28. Kluge R, Sattler B, Seese A, Knapp WH. Attenuation correction by simultaneous emission-transmission myocardial single-photon emission tomography using a technetium-99m-labelled radiotracer: impact on diagnostic accuracy. Eur J Nucl Med. 1997;24:1107-1114. 